\title{
Inhibition of Envelope Correction Method to EMD End Effect
}

\author{
Ma Tianlu \\ Faculty of Information Engineering and Automation \\ Kunming University of Science and Technology \\ Kunming, China \\ mt1117@163.com
}

\author{
Liu Zengli \\ Faculty of Information Engineering and Automation \\ Kunming University of Science and Technology \\ Kunming, China \\ liuzengli@hotmail.com
}

\author{
Zhou Zhi \\ Earthquake Administration of Yunnan Province \\ Kunming, China \\ rocketter6616577@126.com
}

\begin{abstract}
There exists a problem of endpoint effect in EMD, which makes the result of decomposition not perfect. The paper comes up with a kind of envelope correction method to suppress the endpoint effect on this question about periodical signal. The result shows that, the method can solve the problem on distortion of envelope, and improve the accuracy of decomposition.
\end{abstract}

\section{Keywords-EMD;end issue;cyclical;envelope correction}

\section{INTRODUCTION}

In 1998, N.E.Huang [1] and others put forward HHT (Hilbert - Huang Transformation) method, its core is EMD (Empirical Mode Decomposition). EMD serves capability for analyzing non-linear and non-stationary signals for which other traditional methodologies are not applicable. However, due to HHT has not been put forward for long time, so it contains some theoretical problems, such as endpoint effect, that makes the result of decomposition not perfect and further restricts the use of EMD method.

The paper comes up with a kind of envelope correction method to suppress the endpoint effect about periodical signal. Through the analysis of simulated signals to verify the validity of the method.

\section{The EMPIRICAL Mode DeCOMPOSITION MethoD: THE SifTING PROCESS}

According to Huang, the EMD aims at representing an arbitrary signal via a number of intrinsic mode functions (IMFs) and the residual, and an IMF is defined as a function that satisfies the following two conditions:

The number of extrema and the number of zero crossings are either equal or they differ at most by one, together with the mean value of the envelope defined by the local maxima and the envelope of the local minima is zero.

Regarding an arbitrary data series $\mathrm{x}(\mathrm{t})$, using the following algorithm can obtaine the IMFs, shown by Schlurmann[2]:

\section{A. Initialize:}

$$
r_{0}(t)=x(t), i=1
$$

B. Extract the ith IMF:

1) Initialize:

$$
h_{0}(t)=r_{i}(t), k=1
$$

2) Extract the local maxima and minima of $h_{k-1}(t)$.

3) Interpolate the local maxima and the local minima by a cubic spline to form upper and lower envelopes of $h_{k-1}(t)$.

4) Calculate the mean $m_{k-1}(t)$ of the upper and lower envelopes of $h_{k-1}(t)$.

5) Define:

$$
h_{k}(t)=h_{k-1}(t)-m_{k-1}(t)
$$

6)

If IMF criteria are satisfied, then set

$$
I M F_{i}(t)=h_{k}(t)
$$

else go to 2) with $k=k+1$.

C. Define:

$$
r_{i}(t)=r_{i-1}(t)-I M F_{i}(t)
$$

D. If $r_{i}(t)$ still has at least two extrema, then go to (B) with $i=i+1$; else the decomposition is completed and $r_{i}(t)$ is the "residue" of $x(t)$.

Finally, the arbitrary data series $\mathrm{x}(\mathrm{t})$ can be represented by a number of intrinsic mode functions(IMFs) and the residual.The residual represents the average trend of the signal, IMFs show inherent harmonic composition of the signal. 


\section{BASED ON ENVELOPE CORRECTION OF INHIBITION OF EMD END EFFECTS}

\section{A. Basic idea}

EMD endpoint effect manifests as distortion of upper and lower envelope at the endpoint, so the core of settlement the problem is that properly fitting variation tendency of the envelope at the endpoint. The paper[3] mentioned that the development trend of the original signal not only displays at endpoint, but also reappears in signal internal. Especially for the periodic signal, the feature is more obvious, and puts forward a kind of endpoint continuation method which based on waveform matching. To a certain extent, the algorithm can resolve the end effect, but due to continuation signal waveform and the original signal at the endpoint not smooth cohesion can cause "peak" phenomenon, which makes envelope produce error.

In order to further improve the EMD decomposition accuracy, this paper makes use of the idea of the waveform matching, through searching the waveform in the signal internal which is similarity or antisymmetric with wavelet at the endpoint, using the envelope value of the wavelet at the corresponding point instead of envelope value of original signal at the endpoint, then the corrected value regard as new interpolation points, using three Hermite function to fit envelope, in this way, not only can maintain the envelope trend of original signal in the greatest limitation, but also can avoid causing the "peak" phenomenon because of no smooth connection, which can effectively inhibit end effect and improve EMD decomposition accuracy. Based on this idea, this paper proposes envelope correction method to suppress EMD end effect.

\section{B. Envelope correction method}

Set the original signal for $s(t)$, the following to the signal left side analytic process as an example to introduce the envelope correction method:

First step, taking the local maxima (recorded as $m_{0}$ ) and minimum (recorded as $m_{1}$ ) of $s(t)$ as interpolation points, then using piecewise cubic Hermite interpolation method to fit up and low envelope as shown in Figure 1(a).

Second step, taking the waveform of signal $s(t)$ which is from the left endpoint to the second maximum points as the research object, the waveform is recorded as $h_{0}$, and the flip of waveform is recorded as $h_{0}{ }^{\prime}$, both the length are recorded as $L$. Regard the first maximum point as the endpoint reference point which is recorded as $p_{0}$. Recording the length of the left point to the reference point $p_{0}$ as $d_{1}$.

Third step, supposing the set of the maximum value of the signal $s(t)$ are $U_{\max }$, each maxima of $U_{\max }$ as waveform internal reference point(the first maximum and finally maximum except), which are recorded as $p_{i}$. Regarding the point of $\left(p_{i}-\mathrm{d}_{1}\right)$ as the starting point, then taking the subwave which the length is $L$ recorded as $h_{i}$, computing the minimum waveform difference degree of $h_{i}$ with $h_{0}$ and $h_{0}{ }^{\prime}$, respectively.

As can be seen from the formula (6)-(7), the waveform difference degree is $W_{1}$ corresponding to the waveform which is most similarity with the waveform at the endpoint wavelet.The waveform difference degree is $W_{2}$ corresponding to the waveform which is antisymmetry with the waveform at the endpoint wavelet. The waveform difference degree is $W$ recorded as $h_{w}$ which is corresponding to the waveform which is the matchest with the waveform at the endpoint wavelet.

$$
\begin{gathered}
W_{1}=\min \left\{\sum_{t=1}^{t=L}\left\{\left[\mathrm{~h}_{0}(\mathrm{t})+\left(\mathrm{P}_{\mathrm{i}}-\mathrm{P}_{0}\right)\right]-\mathrm{h}_{\mathrm{i}}(\mathrm{t})\right\}^{2}\right\} \\
W_{2}=\min \left\{\sum_{t=1}^{t=L}\left\{\left[\mathrm{~h}_{0}{ }^{\prime}(\mathrm{t})+\left(\mathrm{P}_{\mathrm{i}}-\mathrm{P}_{0}\right)\right]-\mathrm{h}_{\mathrm{i}}(\mathrm{t})\right\}^{2}\right\} \\
W=\min \left[W_{1}, W_{2}\right]
\end{gathered}
$$

Fourth step, recorded the upper envelope of $h_{w}$ as $v$, if $W=W_{1}$, using the value of $v(1)$ instead of the value of upper envelope at the left point, if $W=W_{2}$, then the upper envelope value at the left point is replaced by the value of $v($ end $)$.

At last, taking the value after the correction as a new interpolation point, then using three Hermite function to fit the upper and lower envelope.As shown in Figure 1(b).

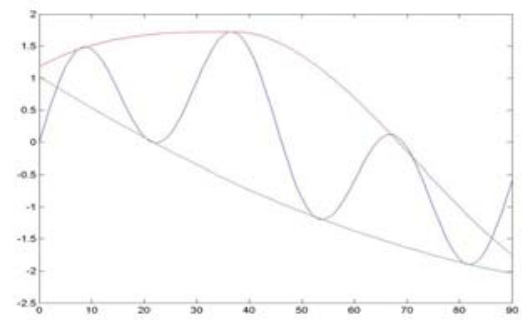

(a) The envelope before correction 


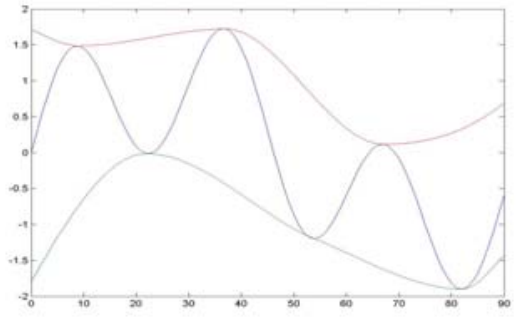

(b) The envelope after correction

Figure 1. Signal envelope

\section{The evaluation index of EMD end effect}

In order to quantitatively describe the effectiveness of envelope correction method of EMD, selecting the following three commonly used indicators as evaluation criteria:

1) The energy were compared before and after EMD[4] as show in formule 9.

$$
\theta=\frac{\left|\sqrt{\sum_{i=1}^{n} R M S_{i}^{2}}-R M S_{\text {orginal }}\right|}{R M S_{\text {orginal }}}
$$

In the formula, the $R M S_{\text {orginal }}$ is the original signal's valid value, the $R M S_{i}$ is the ith's valid value, $n$ is the total number of imf (including EMD's residual item). According to the definition, the smaller the value of $\theta$, the smaller the impact of the end effect, if $\theta=0$, indicates that the end effect did not affect.

2) Compare the correlation coefficient of every IMF component and corresponding component of the original signal [5].

3) Comparison of operation time in order to avoid the complexity of algorithm [6].

\section{Experimental analyses}

In order to test the validity of the envelope correction method, two sets of simulated signal are selected to empirical mode decomposition.

The first simulation signal:

$$
\begin{aligned}
& X(t)=x 1+x 2 ; t \in[0,1] \\
& x 1=3 \sin (2 \pi 100 t)
\end{aligned}
$$

$$
x 2=2 \cos (2 \pi 50 t)
$$

The second simulation signal:

$$
\begin{array}{r}
Y(t)=y 1+y 2 ; t \in[0,1] \\
y 1=5 \sin (2 \pi 100 t)
\end{array}
$$

$y 2=(3+0.2 \sin (2 \pi 10 t)) \cos (2 \tau 50 t+0.5 \sin (2 \pi 20 t))$

And the sampling frequency is $f_{S}=1024 \mathrm{~Hz}$, the sampling interval is $1 / f s$.

The above two kinds of simulation signal's EMD decomposition and Hilbert spectrum as shown in figure 2 .

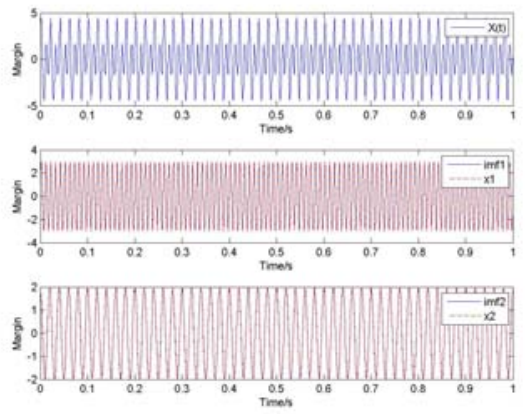

(a) The EMD result of $\mathrm{X}(\mathrm{t})$

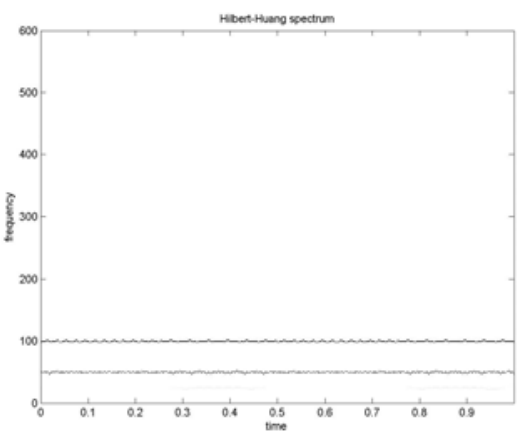

(b) The Hilbert spectrum of $\mathrm{X}(\mathrm{t})$ 


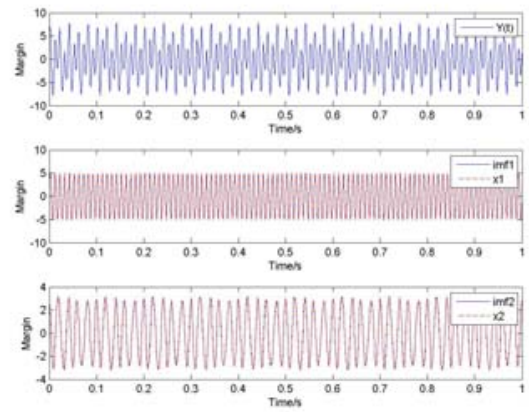

(c) The EMD result of $\mathrm{Y}(\mathrm{t})$

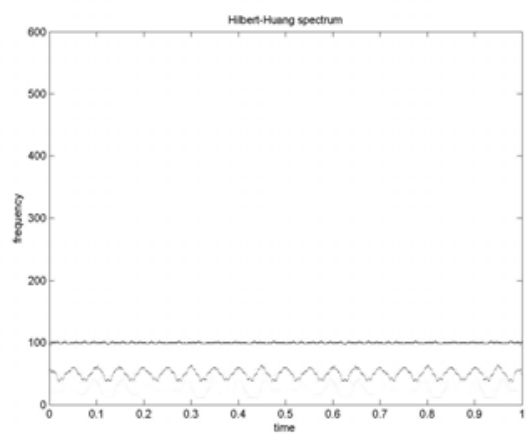

(d) The Hilbert spectrum of $\mathrm{Y}(\mathrm{t})$

Figure 2. The EMD results and Hilbert spectrums after envelope correction method analytic

As can be seen from the figure 2:we get the IMF components which based on the proposed method are really response to the characteristics of original signal, this method intuitively show can effectively suppress the end effect.

In order to quantitatively describe the effectiveness of envelope correction method of EMD, selecting the above introduction three evaluation indexs to compare.

The performance evaluation results which are the above two kinds of simulation signal through different endpoint processing method are shown in Table I.

TABLE I. The PERFormance EVALUATION RESUlts of DifFERENT ENDPOINT PROCESSING METHOD

\begin{tabular}{|c|c|c|c|c|}
\hline \multirow[t]{2}{*}{ Signal } & \multirow{2}{*}{$\begin{array}{l}\text { evaluation } \\
\text { criteria }\end{array}$} & \multicolumn{3}{|c|}{$\begin{array}{c}\text { different } \\
\text { endpoint processing method }\end{array}$} \\
\hline & & $\begin{array}{c}\text { mirror } \\
\text { continuation } \\
\text { method }\end{array}$ & $\begin{array}{l}\text { support } \\
\text { vector } \\
\text { machine }\end{array}$ & $\begin{array}{c}\text { envelope } \\
\text { correction } \\
\text { method }\end{array}$ \\
\hline \multirow{4}{*}{$X(t)$} & $\theta$ & 0.0103 & 0.0402 & 0.0046 \\
\hline & pimf1 & 0.9936 & 0.9936 & 0.9999 \\
\hline & pimf2 & 0.9971 & 0.9971 & 0.9997 \\
\hline & $\mathrm{t}$ & $3 \mathrm{~s}$ & $63 \mathrm{~s}$ & $14 \mathrm{~s}$ \\
\hline \multirow{4}{*}{$\mathrm{Y}(\mathrm{t})$} & $\theta$ & 0.0337 & 0.0358 & 0.0032 \\
\hline & pimf1 & 0.9859 & 0.9859 & 0.9997 \\
\hline & pimf2 & 0.9699 & 0.9680 & 0.9990 \\
\hline & $\mathrm{t}$ & $5 s$ & $134 \mathrm{~s}$ & $22 \mathrm{~s}$ \\
\hline
\end{tabular}

Above results show that the value of $\theta$ and the correlation coefficient which get from envelope correction method are better than the value obtained by mirror extension method and support vector machine, so the envelope correction method which proposed in this paper can effectively inhibit the generation of the end effect, and improve the accuracy of decomposition. But the envelope correction method on the running time is slower than the mirror extension method, which indicates that the program needs to be further optimized.

\section{CONCLUSIONS}

EMD exists a problem of endpoint effect, which makes the result of decomposition distortion. The paper comes up with a kind of envelope correction method to solve the problem. The method is different from the traditional waveform continuation method and extreme value continuation method, which is from envelope distortion angle, chosen the wavelet from the inner signal, which has the most minimum waveform difference degree compared with the ends, using the envelope value of the wavelet at the corresponding point instead of envelope value of original signal at the endpoint. The results show that, the method can solve the problem on distortion of envelope, and improve the accuracy of decomposition.

\section{ACKNOWLEDGMENT}

This work was supported by Natural Science Foundation Project of NSFC under the grant NO.60872157 and NO.61271007.

\section{REFERENCES}

[1] Huang N E,Shen Z,Long S R,Wu M C,Shih H H,Zheng Q.The empirical mode decomposition and the Hilbert spectrum for nonlinear and non-stationary time series analysis.Proceedings of the Royal Society of London,Series A,1998,454(1971): 903-995.

[2] Schlurmann, T., Dose, T., Schimmels, S., 2001. Characteristic modes of the 'Adreanov Tsunami' based on the Hilbert-Huang transformation. In: Proceedings of the 4th International Symposium on Ocean Wave Measurement and Analysis (WAVES 2001). American Society of Civil Engineers (ASCE),vol. 2, pp. 1525-1534.

[3] Shao Chenxi,Wang Jian. An adaptive method for endpoint continuation of EMD[J]. Journal of electronics, 2007, 35 (10):19441948.

[4] Ren Daqian,Wu Zhaorong,Yan Gongbiao. The evaluation index of EMD end effect and the window function method of inhibition end effect [J]. Manufacturing automation, 2007, 29 (1):21-24.

[5] Wang Qiusheng, Duan Danhui. Processing method for end effects of empirical mode decomposition [J]. Computer measurement and control, 2006, (2):1673-1675.

[6] Lin Li, Zhou Ting, Yu Lun. Processing technology for end effects of EMD $[J]$. Computer engineering, 2009, 35 (23) : 265-268. 